\title{
Device for implantation of long-term transvenous cardiac pacemaker electrodes
}

\author{
P. K. CAVES, J. S. GEDDES, and M. E. SCOT T \\ The Cardiac and Thoracic Units, Royal Victoria Hospital, Belfast, Northern Ireland
}

\begin{abstract}
General anaesthesia is usually recommended for the most painful part of long-term transvenous pacemaker implantation, viz., the pulling subcutaneously of the end of the electrode from the neck incision to the site of pacemaker implantation. The ability to perform this step, and therefore the whole procedure, under local anaesthesia is an advantage. We describe a simple, inexpensive device which permits implantation of long-term transvenous cardiac pacemaker electrodes under local anaesthesia. Its use reduces operating time, pain and bleeding and avoids the dangers of general anaesthesia. The device and our implantation technique, as used in 58 patients, are described in detail. The device consists of a solid steel rod introducer and a slightly modified Mousseau Barbin tube (Porges 5870).
\end{abstract}

Long-term pacing from an implanted pacemaker is usually indicated in symptomatic patients with chronic heart block. When using transvenous electrodes, general anaesthesia is recommended and is usually induced after the electrode tip has been passed from the external jugular vein to the apex of the right ventricle. The subcutaneous pocket for the pacemaker is then formed and the pacemaker end of the electrode is pulled through from the neck to the pocket with a long pair of forceps introduced subcutaneously (Harris, 1969; Siddons, 1968).

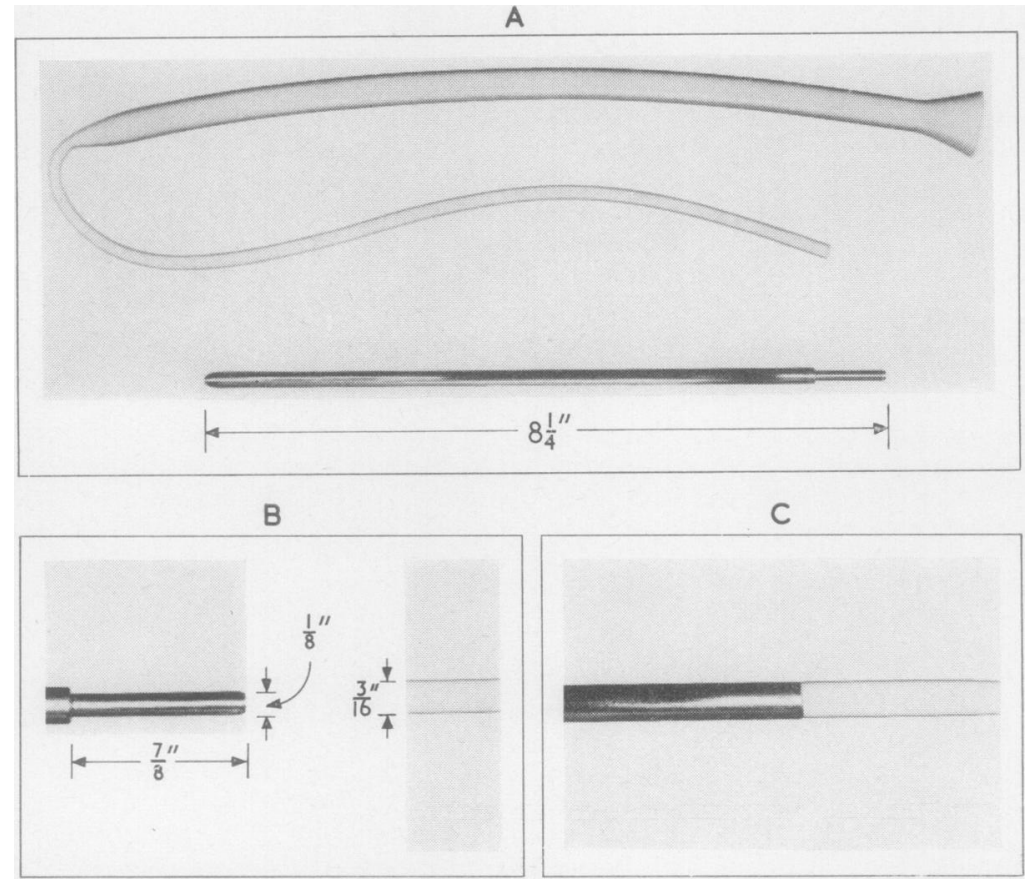

FIG. 1. (A) Modified Mousseau Barbin tube and introducer. (B and $C$ ) End of introducer and tube before and after connection. 
We have not found general anaesthesia necessary during transvenous pacemaker implantation and consider its use hazardous. In addition to its usual risk in the elderly, patients with complete heart block may develop asystole during anaesthetic induction. Using local anaesthesia, the only step that was formerly difficult and painful was drawing down the end of the electrode subcutaneously. The end is blunt and irregular and to pull it through required the formation of a broad subcutaneous tunnel with the forceps. Bleeding in the tunnel occasionally occurred and was difficult to control. Post-operative haematoma increased the risk of infection. In addition the teeth of the forceps sometimes damaged the electrode insulation.

\section{MATERIAL AND METHOD}

We have devised a device that enables this step to be accomplished easily and painlessly under local anaesthesia in a few seconds with minimal bleeding, and with no risk of damage to the electrode. This consists of a stainless steel rod and a slightly modified size 10 Mousseau Barbin tube ${ }^{1}$ (Fig. 1A). The rod is circular in cross section, $3 / 16$ in. ( $5 \mathrm{~mm}$.) diameter and $8 \frac{1}{4}$ in. $(21 \mathrm{~cm}$.) long. One end is tapered off and the other is adapted to fit firmly and flush with the 'tail' of the Mousseau Barbin tube (Fig. 1, B and C). The 'funnel' end of the tube is cut away until the end of the electrode just fits inside (Fig. 2).

Our technique following the transvenous passage of the electrode into the right ventricle is as follows:

Using $1 \%$ xylocaine with adrenaline $1 / 200,000$, an area of skin in the axilla is anaesthetized and an adequate horizontal incision is made. The subcutaneous pocket is formed by dissection. A long needle is introduced from the axilla to the neck and a 'track' of subcutaneous tissue is anaesthetized with the local anaesthetic. The metal rod with 'tail' attached is introduced from the neck, passed down the subcutaneous track to the axilla and pulled through, leaving the 'tail' lying subcutaneously.

The electrode is then disconnected from the temporary pacemaker and attached to the funnel of the tube with a strong silk suture, and the tube and electrode are pulled through smoothly (Fig. 2). The suture is cut and the end of the electrode is connected to the permanent pacemaker, which is then implanted in the axillary pocket.

We have now used this device 58 times for the implantation of various types of long-term transvenous cardiac pacemaker electrodes. The metal rod was easily manufactured from stainless steel rod in the hospital workshop. Mousseau Barbin tubes are available in any thoracic unit. Both can be sterilized and used repeatedly.

1 Porges 5870
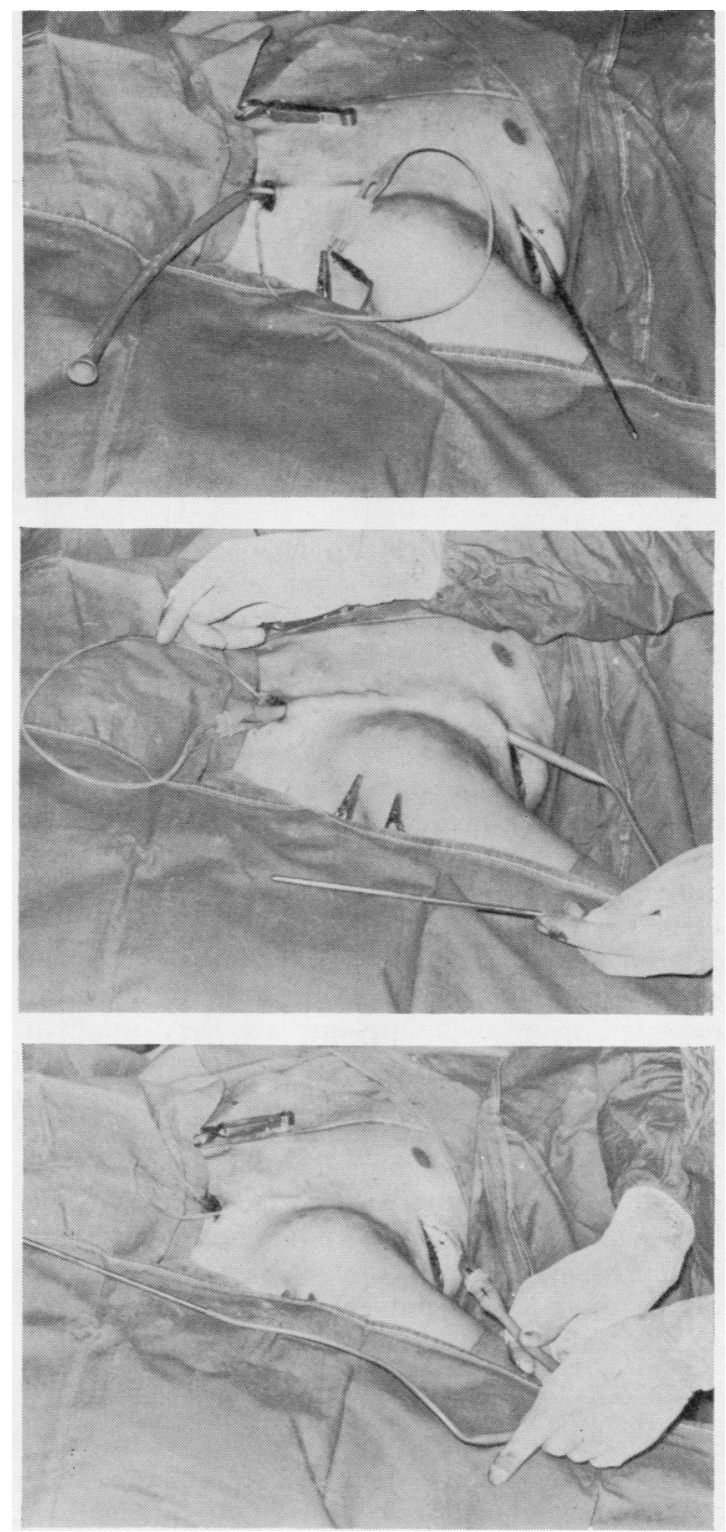

FIG. 2. Use of device for implantation of American Optical Electrode (American Optical Chardack Electrode, Catalogue No. 283104).

We wish to thank Mr. M. Kennedy for his help in constructing the steel introducer and Miss P. Haddock for typing the manuscript.

\section{REFERENCES}

Harris, A. M. (1969). Transvenous pacing. Brit. J. Hosp. Med., 2, 1131. Siddons, H. (1968). Cardiac Pacing in Operative Surgery, edited by
C. Rob and R. Smith, Vol. 2, p. 336 . 